\title{
Bounded Traveling Waves of the (2+1)-Dimensional Zoomeron Equation
}

\author{
Yuqian Zhou, ${ }^{1}$ Shanshan Cai, ${ }^{1}$ and Qian Liu ${ }^{2}$ \\ ${ }^{1}$ School of Applied Mathematics, Chengdu University of Information Technology, Chengdu, Sichuan 610225, China \\ ${ }^{2}$ School of Computer Science and Technology, Southwest University for Nationalities, Chengdu, Sichuan 610041, China \\ Correspondence should be addressed to Yuqian Zhou; cs97zyq@aliyun.com
}

Received 5 December 2014; Accepted 24 January 2015

Academic Editor: P. Balasubramaniam

Copyright (c) 2015 Yuqian Zhou et al. This is an open access article distributed under the Creative Commons Attribution License, which permits unrestricted use, distribution, and reproduction in any medium, provided the original work is properly cited.

\begin{abstract}
The bifurcation method of dynamical system and numerical simulation method of differential equation are employed to investigate the $(2+1)$-dimensional Zoomeron equation. We obtain the parameter bifurcation sets that divide the parameter space into different regions which correspond to qualitatively different phase portraits. According to these phase portraits, all bounded traveling waves are identified and simulated, including solitary wave solutions, shock wave solutions, and periodic wave solutions. Furthermore, all exact expressions of these bounded traveling waves are given. Among them, the elliptic function periodic wave solutions are new solutions.
\end{abstract}

\section{Introduction}

In this paper, we consider the following $(2+1)$-dimensional Zoomeron equation [1]:

$$
\left(\frac{U_{x y}}{U}\right)_{t t}-\left(\frac{U_{x y}}{U}\right)_{x x}+2\left(U^{2}\right)_{x t}=0
$$

where function $U(t, x, y)$ is the amplitude of the relevant wave mode. This highly nonlinear equation plays an important role in describing the evolution of a single scalar field and is a convenient model to display the novel phenomena associated with boomerons and trappons. If $y=t$, the $(2+1)$-dimensional Zoomeron equation reduces to its $(1+1)$ dimensional form which can be regarded as a subcase of the boomeron equation essentially.

In recent decades, the investigation of traveling wave solutions for nonlinear partial differential equation is an active area in mathematics and physics. It is significant to seek new solutions of a PDE, since they may provide more information to understand the complicated wave phenomena and find new properties. The traveling wave solutions of (1) have been focused on by many authors. Various direct methods have been applied to obtain its exact traveling wave solutions. Firstly, Abazari [2] used $\left(G^{\prime} / G\right)$-expansion method to obtain three types of exact solutions of (1), including hyperbolic function solutions, trigonometric function solutions, and rational function solutions. In 2012, Alquran and AlKhaled [3] discussed four types of soliton solutions of the equation by using some direct mathematical methods, such as the extended tanh function method, the exponential function method, and the $\operatorname{sech}^{p}-\tanh ^{p}$ function method. In 2013, Irshad and Mohyud-Din [4] applied the tanh-coth method to give several solitary wave solutions. Later, Qawasmeh [5] obtained two types of periodic solutions by using the sinecosine function method. Recently, Khan and Ali Akbar [6] got some new solutions with the modified simple equation (MSE) method.

Though there have been so many profound results about traveling wave solutions of (1) which contributed to our understanding of nonlinear physical phenomena and wave propagation, some traveling wave solutions could be still lost because of the defects of the direct methods caused by the auxiliary equations and the hypotheses about the forms of solutions. In addition, the direct methods can not clearly explain how these solutions evolve when the parameters vary, although they can be used to obtain solutions concisely and efficiently. These problems arouse our great interest in surveying the traveling wave system of (1) again. In fact, 
it has involved bifurcation of traveling wave solutions. In general, three basic types of bounded traveling waves could occur for a PDE, which are periodic waves, shock waves, and solitary waves. Recall that heteroclinic orbits are trajectories which have two distinct equilibria as their $\alpha$ - and $\omega$-limit sets and homoclinic orbits are trajectories whose $\alpha$ - and $\omega$-limit sets consist of the same equilibrium. So, the three basic types of bounded traveling waves mentioned above correspond to periodic, heteroclinic, and homoclinic orbits of the traveling wave system of a PDE, respectively $[7,8]$. It is just the relationship that makes the bifurcation theory of dynamical system become an effective method to investigate bounded traveling waves of a PDE. In recent decades, many efforts have been devoted to bifurcations of traveling waves of PDEs. In 1997, Peterhof et al. [9] investigated persistence and continuation of exponential dichotomies for solitary wave solutions of semilinear elliptic equations on infinite cylinders so that Lyapunov-Schmidt reduction can be applied near solitary waves. Sánchez-Garduño and Maini [10] considered the existence of one-dimensional traveling wave solutions in nonlinear diffusion degenerate Nagumo equations and employed a dynamical systems approach to prove the bifurcation of a heteroclinic cycle. Later, Katzengruber et al. [7] analyzed the bifurcation of traveling waves such as Hopf bifurcation, multiple periodic orbit bifurcation, homoclinic bifurcation, and heteroclinic bifurcation in a standard model of electrical conduction in extrinsic semiconductors, which in scaled variables is actually a singular perturbation problem of a 3-dimensional ODE system. In 2002, Constantin and Strauss [11] constructed periodic traveling waves with vorticity for the classical inviscid water wave problem under the influence of gravity, described by the Euler equation with a free surface over a flat bottom, and used global bifurcation theory to construct a connected set of such solutions, containing flat waves as well as waves that approach flows with stagnation points. In 2003, Huang et al. [12] employed the Hopf bifurcation theorem to established the existence of traveling front solutions and small amplitude traveling wave train solutions for a reaction-diffusion system based on a predator-prey model, which are equivalent to heteroclinic orbits and small amplitude periodic orbits in $\mathbb{R}^{4}$, respectively. Besides, many results on bifurcations of traveling waves for Burgers-Huxley equation, RLW-Burgers equation, Davey-Stewartson-type equations, and microstructured solid model can be found from [13-16].

Motivated by the reasons above, we try to seek all bounded traveling waves of the $(2+1)$-dimensional Zoomeron equation and investigate their bifurcation behavior. By using the bifurcation methods of dynamical system [17-20] and the numerical simulation method of differential equation, we obtain the parameter bifurcation sets that divide the parameter space into different regions which correspond to qualitatively different phase portraits. According to the phase portraits, all possible bounded traveling waves are identified and simulated including solitary waves, shock waves, and periodic waves. Furthermore, by calculating some complicated elliptic integrals, we obtain the exact expressions of these bounded traveling waves. Among them, the elliptic function periodic wave solutions are new solutions.

\section{Traveling Wave System and Bifurcation Analysis}

Letting $U(x, y, t)=u(\xi)=u(x+a y-c t)$, we transform (1) into its corresponding traveling wave system as follows:

$$
c^{2}\left(\frac{a u^{\prime \prime}}{u}\right)^{\prime \prime}-\left(\frac{a u^{\prime \prime}}{u}\right)^{\prime \prime}-2 c\left(u^{2}\right)^{\prime \prime}=0,
$$

where ' denotes $d / d \xi$ and $c \neq 0$ is the velocity of wave. Integrating (2) twice and omitting a free term, we get

$$
\frac{a c^{2} u^{\prime \prime}}{u}-\frac{a u^{\prime \prime}}{u}-2 c u^{2}=e,
$$

where parameter $e$ is the integral constant. By (3), $u^{\prime \prime}$ can be solved by

$$
u^{\prime \prime}=\frac{2 c u^{3}+e u}{a\left(c^{2}-1\right)} .
$$

Then, (4) can be transformed into the following planner dynamical system

$$
\begin{aligned}
& u^{\prime}=y, \\
& y^{\prime}=\frac{2 c u^{3}+e u}{a\left(c^{2}-1\right)},
\end{aligned}
$$

which has the first integral

$$
H(u, y)=y^{2}-\frac{c u^{4}+e u^{2}}{a\left(c^{2}-1\right)},
$$

where $a\left(c^{2}-1\right) \neq 0$.

Next, we study the distribution and properties of critical points of system (5).

Theorem 1. When e/c $<0$, system (5) has three critical points $A(0,0), B(\sqrt{-e / 2 c}, 0)$, and $C(-\sqrt{-e / 2 c}, 0)$. If $e / a\left(c^{2}-1\right)>0$, the critical point $A$ is a saddle point, while $B$ and $C$ are centers. If e/ $a\left(c^{2}-1\right)<0$, the critical point $A$ is a center, while $B$ and $C$ are saddle points.

When $e / c>0$, system (5) has only one critical point $A(0,0)$. If e/a $\left(c^{2}-1\right)>0, A$ is a saddle point. If $e / a\left(c^{2}-1\right)<0$, then $A$ is a center.

Whene $=0$, system (5) has a unique critical point of higher $\operatorname{order} A(0,0)$. If $c / a\left(c^{2}-1\right)>0, A$ is a saddle point. If $c / a\left(c^{2}-\right.$ $1)<0$, then $A$ is a center.

Proof. When $e / c<0$, by a direct computation, one can check that system (5) has three critical points $A(0,0), B(\sqrt{-e / 2 c}, 0)$, and $C(-\sqrt{-e / 2 c}, 0)$. Let $M(u, y)$ denote the coefficient matrix of the linearized system of $(5)$ at point $(u, y)$. Then,

$$
\begin{aligned}
& M(A)=\left[\begin{array}{cc}
0 & 1 \\
\frac{e}{a\left(c^{2}-1\right)} & 0
\end{array}\right], \\
& M(B)=M(C)=\left[\begin{array}{cc}
0 & 1 \\
\frac{-2 e}{a\left(c^{2}-1\right)} & 0
\end{array}\right] .
\end{aligned}
$$




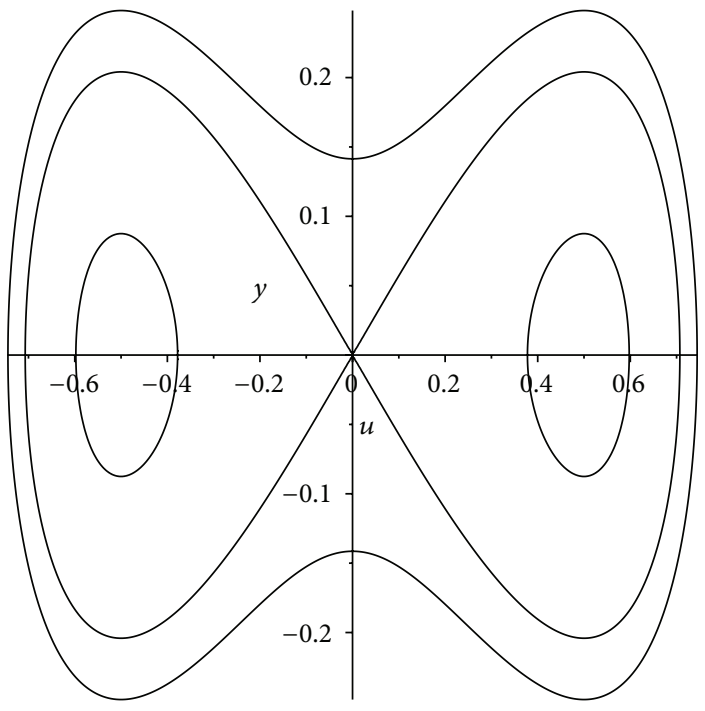

(a) $c=-2, e=1$, and $a=1$

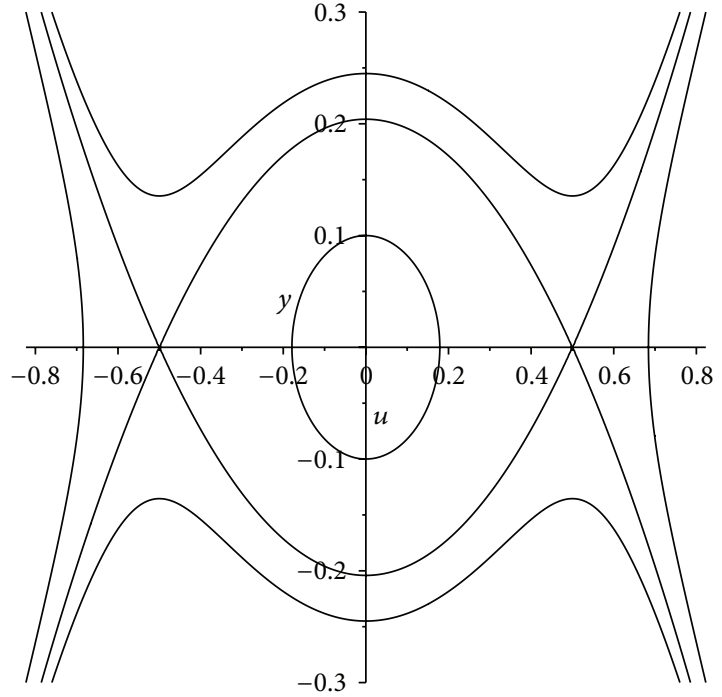

(b) $c=-2, e=1$, and $a=-1$

FIGURE 1: The phase portraits of system (5) for $e / c<0$.

If $e / a\left(c^{2}-1\right)>0$, one can check that the coefficient determinants

$$
\begin{aligned}
& \operatorname{det} M(A)=-\frac{e}{a\left(c^{2}-1\right)}<0, \\
& \operatorname{det} M(B)=\operatorname{det} M(C)=\frac{2 e}{a\left(c^{2}-1\right)}>0 .
\end{aligned}
$$

By the theory of planner dynamical system [17-20], the critical point $A$ is a saddle point, while $B$ and $C$ are centers. If $e / a\left(c^{2}-1\right)<0$, we have

$$
\begin{aligned}
& \operatorname{det} M(A)=-\frac{e}{a\left(c^{2}-1\right)}>0, \\
& \operatorname{det} M(B)=\operatorname{det} M(C)=\frac{2 e}{a\left(c^{2}-1\right)}<0 .
\end{aligned}
$$

It implies that the critical point $A$ is a center, while $B$ and $C$ are saddle points.

Similar analysis can be employed to prove Case 2. Here, we omit it for simplicity.

When $e=0$, a direct computation shows system (5) has a unique critical point $A(0,0)$ with a degenerated coefficient matrix

$$
M(A)=\left[\begin{array}{ll}
0 & 1 \\
0 & 0
\end{array}\right] .
$$

It means that $A$ is a critical point of higher order. In fact, when $e=0$, system (5) has the form

$$
\begin{aligned}
& u^{\prime}=y=P(u, y), \\
& y^{\prime}=\frac{2 c u^{3}}{a\left(c^{2}-1\right)}=Q(u, y) .
\end{aligned}
$$

By the qualitative theory of differential equation [20, Theorem 7.2, Chapter 2], we have $a_{2 m+1}=2 c / a\left(c^{2}-1\right)$ and $b_{n}=0$. It implies that $A$ is a saddle point if $c / a\left(c^{2}-1\right)>0$, while it is a center (or focus) if $c / a\left(c^{2}-1\right)<0$. Furthermore, we note that $P(-u, y)=P(u, y)$ and $Q(u,-y)=-Q(u, y)$. It means that the vector field is symmetric and $A$ is a center if $c / a\left(c^{2}-1\right)<0$.

Based on the analysis of the critical points and the properties of the Hamiltonian system, we obtain the parameter bifurcation sets for system (5) as follows.

Case 1. Consider $e / c<0$ and $e / a\left(c^{2}-1\right)>0$. There exist two homoclinic orbits $\gamma^{B}$ (the right one) and $\gamma^{C}$ (the left one), which connect the saddle $A$. Centers $B$ and $C$ are surrounded, respectively, by the families of periodic orbits

$$
\begin{aligned}
& \Gamma_{1}^{B}(h):=\left\{H(u, y)=h, h \in\left(\frac{e^{2}}{4 a c\left(c^{2}-1\right)}, 0\right)\right\}, \\
& \Gamma_{1}^{C}(h):=\left\{H(u, y)=h, h \in\left(\frac{e^{2}}{4 a c\left(c^{2}-1\right)}, 0\right)\right\} .
\end{aligned}
$$

Moreover, $\Gamma_{1}^{B}(h)$ (resp., $\left.\Gamma_{1}^{C}(h)\right)$ tends to $B$ (resp., $C$ ) as $h \rightarrow$ $e^{2} / 4 a c\left(c^{2}-1\right)$ and tends to $\gamma^{B}$ (resp., $\left.\gamma^{C}\right)$ as $h \rightarrow 0$, as shown in Figure 1(a).

Case 2. Consider $e / c<0$ and $e / a\left(c^{2}-1\right)<0$. There exist two heteroclinic orbits $\gamma_{U}^{A}$ (the upper one) and $\gamma_{L}^{A}$ (the lower one), which connect the saddles $B$ and $C$. Center $A$ is surrounded by the family of periodic orbits

$$
\Gamma_{2}^{A}(h):=\left\{H(u, y)=h, h \in\left(0, \frac{e^{2}}{4 a c\left(c^{2}-1\right)}\right)\right\} .
$$




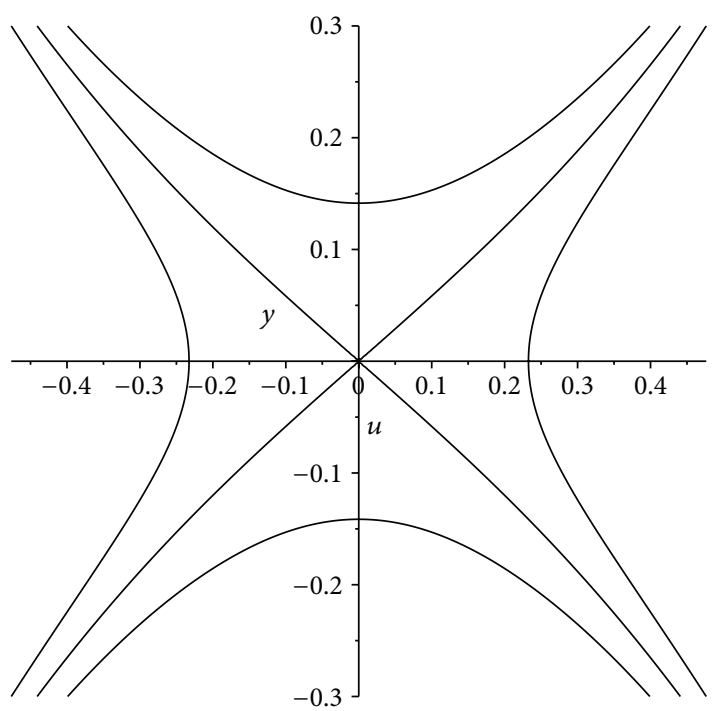

(a) $c=2, e=1$, and $a=1$

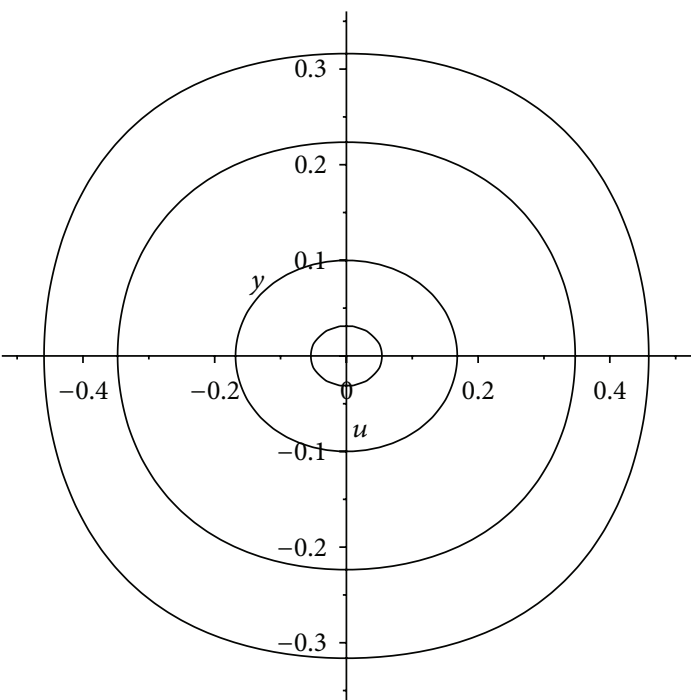

(b) $c=2, e=1$, and $a=-1$

FIgURE 2: The phase portraits of system (5) for $e / c>0$.

Moreover, $\Gamma_{2}^{A}(h)$ tends to center $A$ as $h \rightarrow 0$ and tends to $\gamma_{U}^{A}$ and $\gamma_{L}^{A}$ as $h \rightarrow e^{2} / 4 a c\left(c^{2}-1\right)$, as shown in Figure 1(b).

Case 3. Consider $e / c>0$. There exist no bounded orbits of system (5) if $e / a\left(c^{2}-1\right)>0$, as shown in Figure 2(a). There exist a family of periodic orbits if $e / a\left(c^{2}-1\right)<0$, as shown in Figure 2(b).

Case 4. Consider $e=0$. There exist no bounded orbits of system (5) if $c / a\left(c^{2}-1\right)>0$, as shown in Figure 3(a). There exist a family of periodic orbits if $c / a\left(c^{2}-1\right)<0$, as shown in Figure 3(b).

From the phase portraits, all bounded orbits can be identified clearly as follows:

(1) the closed orbits (see Figures 1(a), 1(b), 2(b), and 3(b)),

(2) the homoclinic orbits (see Figure 1(a)),

(3) the heteroclinic orbits (see Figure 1(b)).

From the relations between the bounded orbits and the bounded traveling waves mentioned in Section 1, all bounded traveling waves can be simulated. Taking Figure 1(a), for example, we can choose $u(0)=0.2$ and $u^{\prime}(0)=0$ as an initial value to simulate a periodic wave of (1) as shown in Figure 4(a), which corresponds to a closed orbit in $\Gamma_{1}^{B}(h)$. Similarly, letting $u(0)=-0.2$ and $u^{\prime}(0)=0$ as an initial value, we can simulate another periodic wave shown in Figure 4(b), which corresponds to a closed orbit in $\Gamma_{1}^{C}(h)$.

Similar method can be employed to simulate solitary waves (see Figures 5(a) and 5(b)) and shock waves (see Figures 6(a) and 6(b)) which correspond to the homoclinic orbits and the heteroclinic orbits, respectively.

\section{The Explicit Expressions of Bounded Traveling Waves}

In this section, we will give the explicit expressions of all bounded traveling waves of system (1).

3.1. The Periodic Wave Solutions. Consider the case $e / c<0$ and $e / a\left(c^{2}-1\right)<0$. In this case, system (5) has a family of periodic orbits $\Gamma_{2}^{A}(h)$. By (6), one of the closed orbits $\Gamma_{2}^{A}(h)$ can be expressed by

$$
y= \pm \sqrt{\frac{c}{a\left(c^{2}-1\right)}} \sqrt{(u-p)(u-q)(r-u)(s-u)},
$$

where $p, q, r$, and $s$ are reals and $p<q<u<r<s$. We suppose that the period of the closed orbit is $2 T$ and choose $u(0)=q$. From (5) and (14), we have

$$
\begin{aligned}
& \int_{q}^{u} \sqrt{\frac{a\left(c^{2}-1\right)}{c}} \frac{d u}{\sqrt{(u-p)(u-q)(r-u)(s-u)}} \\
& \quad=\int_{0}^{\xi} d \xi, \quad 0<\xi<T, \\
& -\int_{u}^{q} \sqrt{\frac{a\left(c^{2}-1\right)}{c}} \frac{d u}{\sqrt{(u-p)(u-q)(r-u)(s-u)}} \\
& =\int_{\xi}^{0} d \xi, \quad-T<\xi<0,
\end{aligned}
$$




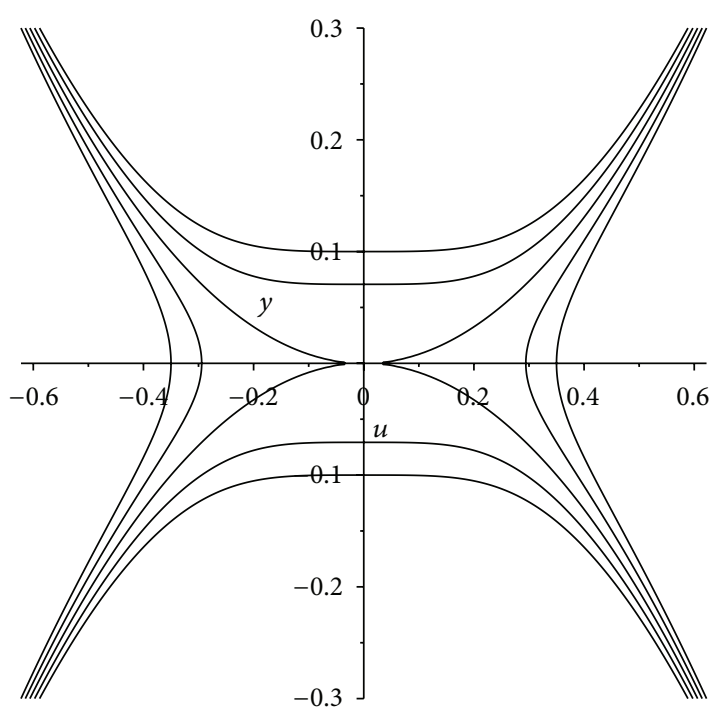

(a) $c=2, e=0$, and $a=1$

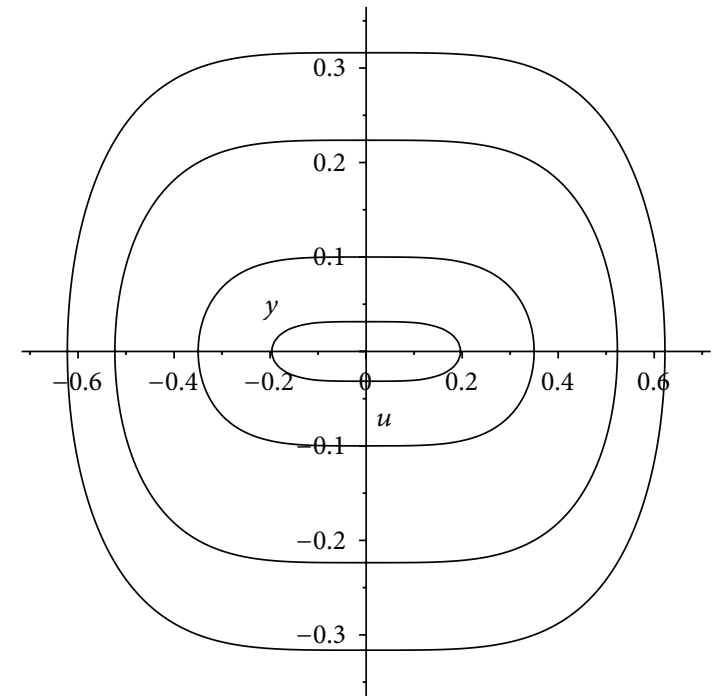

(b) $c=2, e=0$, and $a=-1$

FIGURE 3: The phase portraits of system (5) for $e=0$.

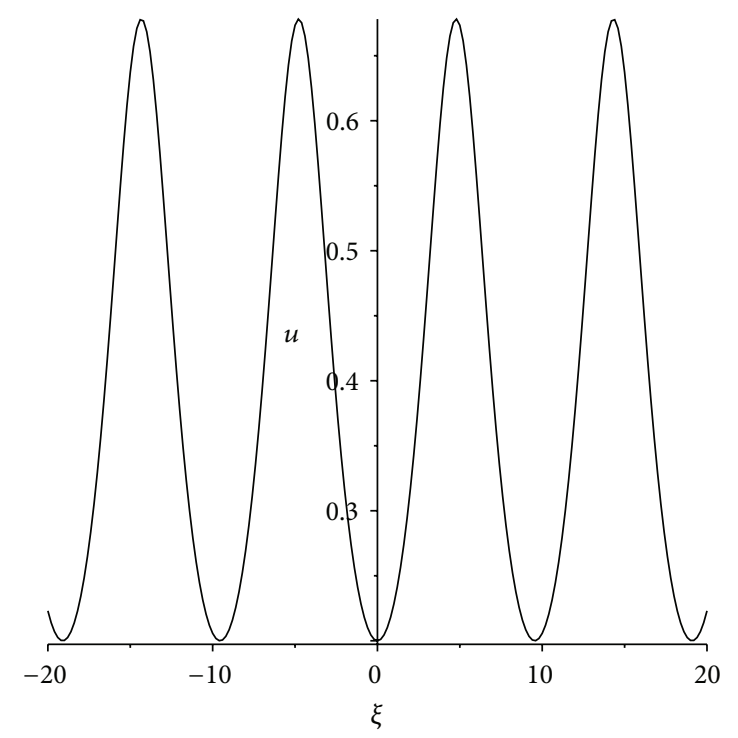

(a) $u(0)=0.2$ and $u^{\prime}(0)=0$

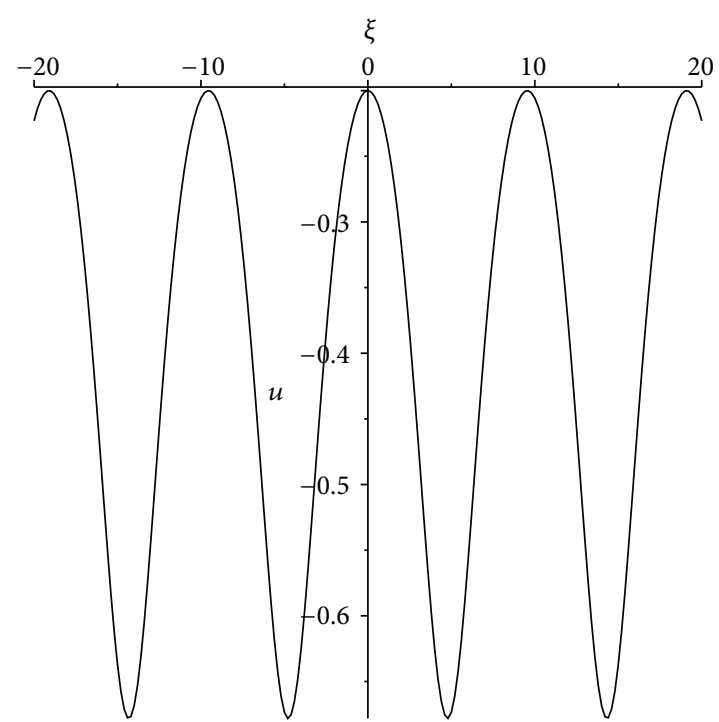

(b) $u(0)=-0.2$ and $u^{\prime}(0)=0$

FIgURE 4: The simulations of the periodic waves.

which can be rewritten as

$$
\int_{q}^{u} \sqrt{\frac{a\left(c^{2}-1\right)}{c}} \frac{d u}{\sqrt{(u-p)(u-q)(r-u)(s-u)}}=|\xi| .
$$

By calculating the elliptic integral

$$
\begin{aligned}
& \int_{q}^{u} \frac{d u}{\sqrt{(u-p)(u-q)(r-u)(s-u)}} \\
& \quad=g \operatorname{sn}^{-1}\left(\sqrt{\frac{(r-p)(u-q)}{(r-q)(u-p)}}, k\right),
\end{aligned}
$$

we get the corresponding periodic wave solution

$$
\begin{aligned}
u(\xi)=p+ & ((r-p)(q-p)) \\
& \cdot\left((r-p)-(r-q) \mathrm{sn}^{2}\right. \\
& \left.\cdot\left(\sqrt{\frac{c}{a\left(c^{2}-1\right)}} \frac{\sqrt{(s-q)(r-p)}}{2}|\xi|\right)\right)^{-1},
\end{aligned}
$$






(a) $u(0)=0.707106739$ and $u^{\prime}(0)=0$

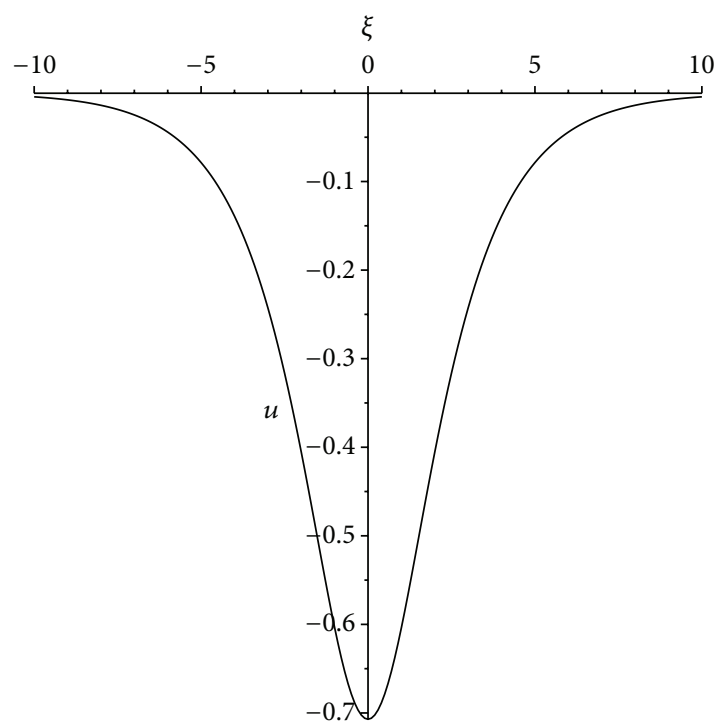

(b) $u(0)=-0.707106739$ and $u^{\prime}(0)=0$

FIGURE 5: The simulations of the solitary waves.

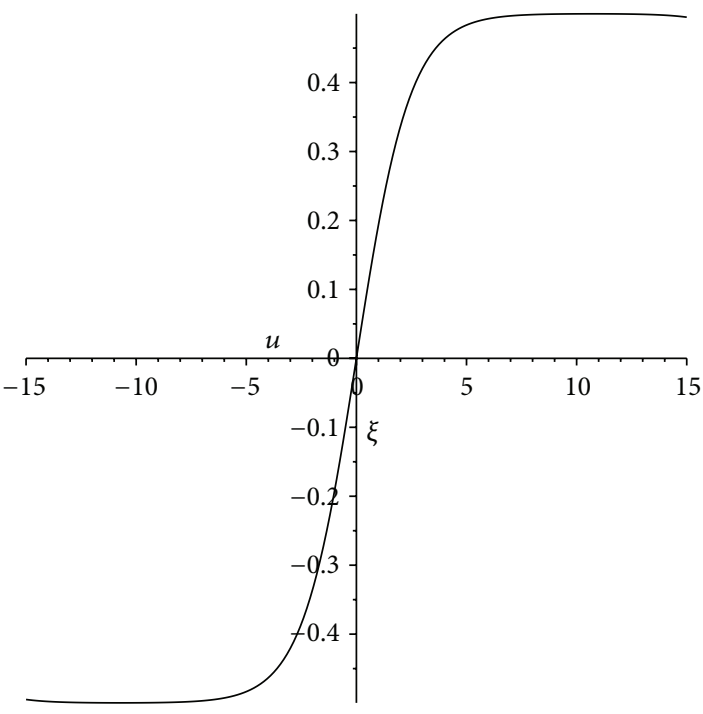

(a) $u(0)=0$ and $u^{\prime}(0)=0.204123974$

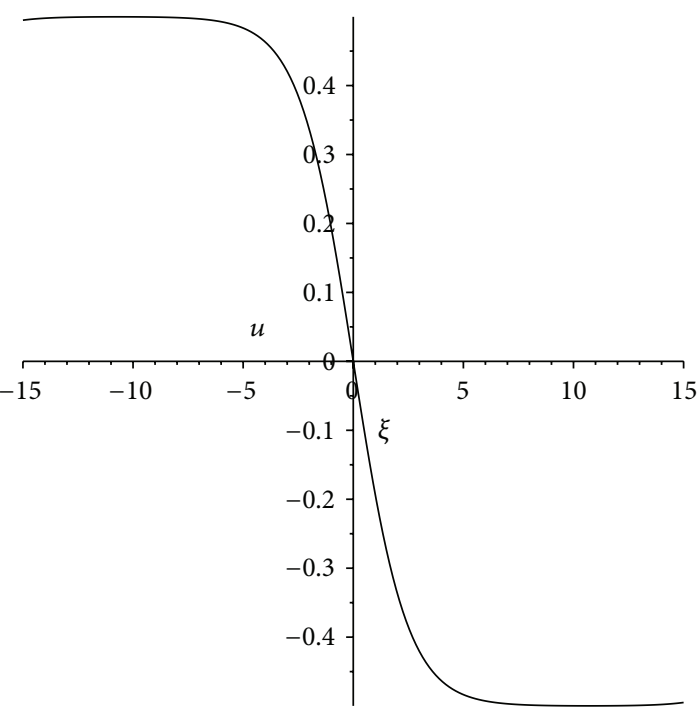

(b) $u(0)=0$ and $u^{\prime}(0)=-0.204123974$

Figure 6: The simulations of the shock waves.

shown in Figure $7(\mathrm{a})$, where $g=2 / \sqrt{(s-q)(r-p)}$ and $k^{2}=$ $(r-q)(s-p) /(s-q)(r-p)$.

Next, consider the case $e / c<0$ and $e / a\left(c^{2}-1\right)>0$. In this case, system (5) has two families of periodic orbits $\Gamma_{1}^{C}(h)$ and $\Gamma_{1}^{B}(h)$. By (6), one of the closed orbits $\Gamma_{1}^{C}(h)$ has the form

$$
y= \pm \sqrt{-\frac{c}{a\left(c^{2}-1\right)}} \sqrt{(u-p)(q-u)(r-u)(s-u)},
$$

where $p<u<q<r<s$. We suppose that the period of the closed orbit is $2 T_{1}$ and choose $u(0)=p$. According to (5) and (19), we have

$$
\begin{aligned}
& \int_{p}^{u} \sqrt{-\frac{a\left(c^{2}-1\right)}{c}} \frac{d u}{\sqrt{(u-p)(q-u)(r-u)(s-u)}} \\
& \quad=\int_{0}^{\xi} d \xi, \quad 0<\xi<T_{1},
\end{aligned}
$$




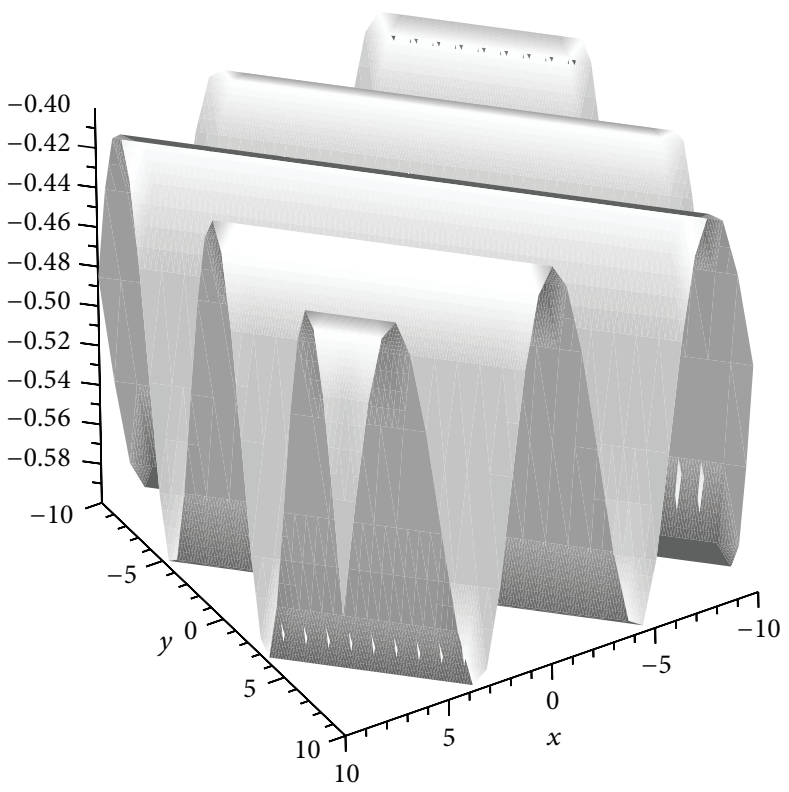

(a)

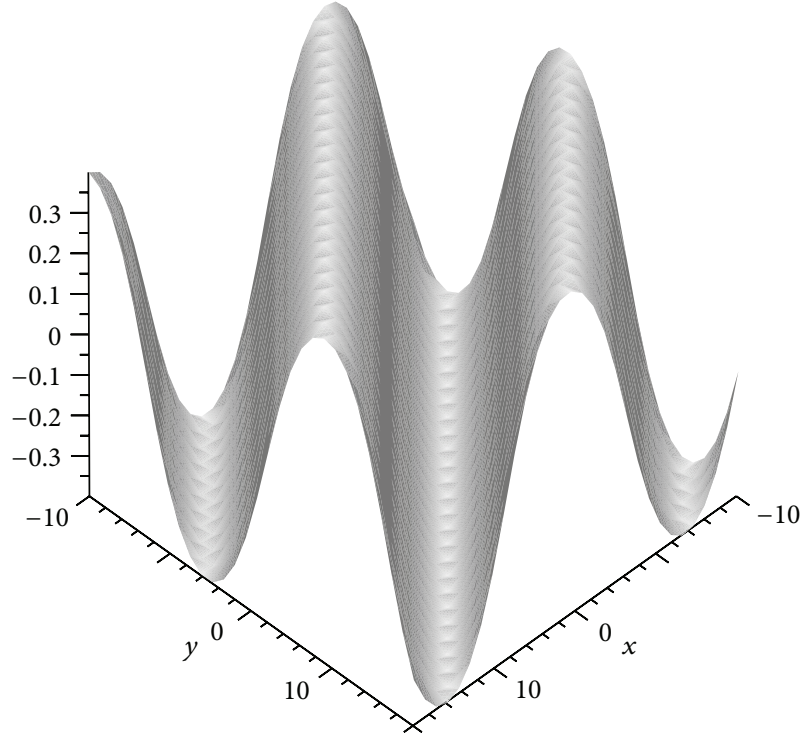

(b)

FIGURE 7: The periodic wave solutions.

$$
\begin{aligned}
& -\int_{u}^{p} \sqrt{-\frac{a\left(c^{2}-1\right)}{c}} \frac{d u}{\sqrt{(u-p)(q-u)(r-u)(s-u)}} \\
& =\int_{\xi}^{0} d \xi, \quad-T_{1}<\xi<0,
\end{aligned}
$$

which can be rewritten as

$$
\int_{p}^{u} \sqrt{-\frac{a\left(c^{2}-1\right)}{c}} \frac{d u}{\sqrt{(u-p)(q-u)(r-u)(s-u)}}=|\xi| .
$$

By calculating the elliptic integral

$$
\begin{aligned}
& \int_{p}^{u} \frac{d u}{\sqrt{(u-p)(q-u)(r-u)(s-u)}} \\
& \quad=g \operatorname{sn}^{-1}\left(\sqrt{\frac{(s-q)(u-p)}{(q-p)(s-u)}}, k\right),
\end{aligned}
$$

we get the corresponding periodic wave solution

$$
\begin{aligned}
u(\xi)=s & -((s-q)(s-p)) \\
& \left((s-q)+(q-p) s^{2}\right. \\
& \left.\left(\sqrt{-\frac{c}{a\left(c^{2}-1\right)}} \frac{\sqrt{(s-q)(r-p)}}{2}|\xi|\right)\right)^{-1},
\end{aligned}
$$

as shown in Figure 7(b), where $g=2 / \sqrt{(s-q)(r-p)}$ and $k^{2}=(s-r)(q-p) /(s-q)(r-p)$. by

Similarly, one of the closed orbits $\Gamma_{1}^{B}(h)$ can be expressed

$$
y= \pm \sqrt{-\frac{c}{a\left(c^{2}-1\right)}} \sqrt{(u-p)(u-q)(u-r)(s-u)},
$$

where $p<q<r<u<s$. Supposing that the period of the closed orbit is $2 T_{2}$ and choosing $u(0)=r$, we have

$$
\begin{aligned}
& \int_{r}^{u} \sqrt{-\frac{a\left(c^{2}-1\right)}{c}} \frac{d u}{\sqrt{(u-p)(u-q)(u-r)(s-u)}} \\
& \quad=\int_{0}^{\xi} d \xi, \quad 0<\xi<T_{2}, \\
& -\int_{u}^{r} \sqrt{-\frac{a\left(c^{2}-1\right)}{c}} \frac{d u}{\sqrt{(u-p)(u-q)(u-r)(s-u)}} \\
& =\int_{\xi}^{0} d \xi, \quad-T_{2}<\xi<0,
\end{aligned}
$$

which can be combined to

$$
\int_{r}^{u} \sqrt{-\frac{a\left(c^{2}-1\right)}{c}} \frac{d u}{\sqrt{(u-p)(u-q)(u-r)(s-u)}}=|\xi| .
$$




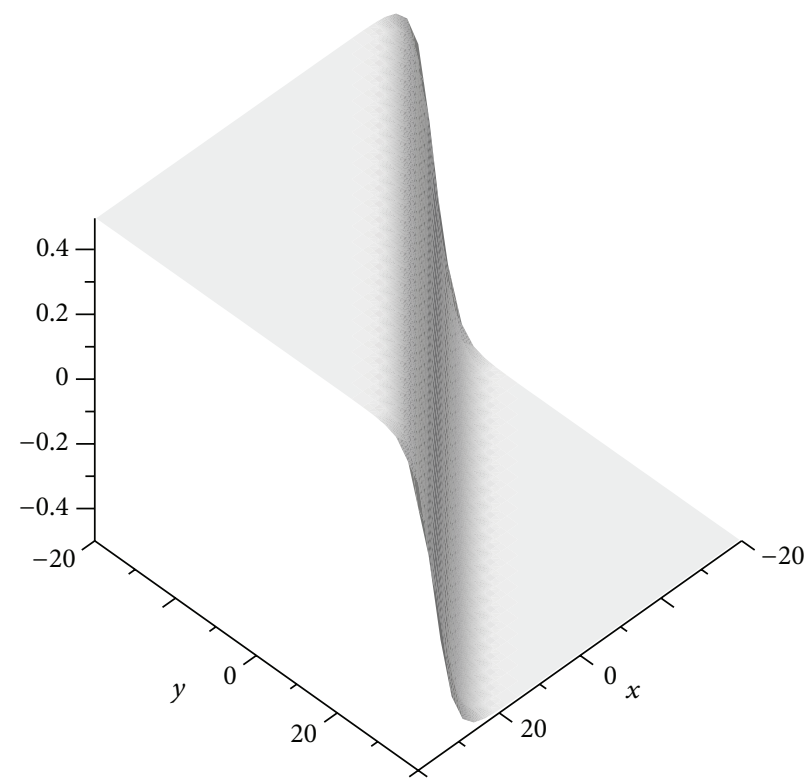

(a)

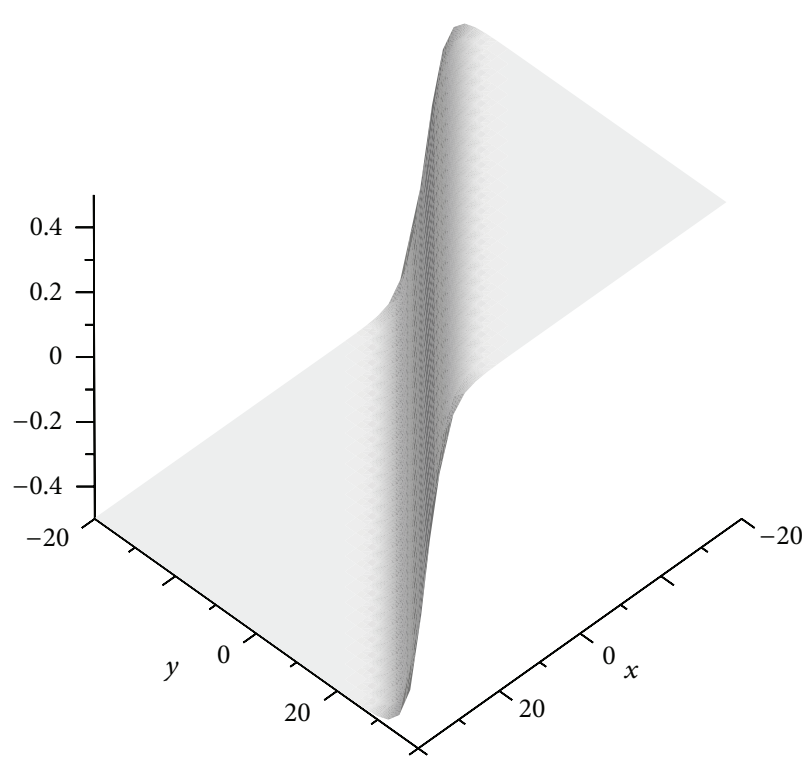

(b)

FIGURE 8: The shock wave solutions.

Noting that

$$
\begin{aligned}
& \int_{r}^{u} \frac{d u}{\sqrt{(u-p)(u-q)(u-r)(s-u)}} \\
& \quad=g \operatorname{sn}^{-1}\left(\sqrt{\frac{(s-q)(u-r)}{(s-r)(u-q)}}, k\right)
\end{aligned}
$$

we get the corresponding periodic wave solution

$$
\begin{aligned}
u(\xi)=q+ & ((r-q)(s-q)) \\
& \cdot\left((s-q)-(s-r) s^{2}\right. \\
& \left.\cdot\left(\sqrt{-\frac{c}{a\left(c^{2}-1\right)}} \frac{\sqrt{(s-q)(r-p)}}{2}|\xi|\right)\right)^{-1},
\end{aligned}
$$

where $g=2 / \sqrt{(s-q)(r-p)}$ and $k^{2}=(s-r)(q-p) /(s-q)(r-$ p).

3.2. The Shock Wave Solutions. In case $e / c<0$ and $e / a\left(c^{2}-\right.$ $1)<0$, the heteroclinic orbits $\gamma_{U}^{A}$ and $\gamma_{L}^{A}$ can be expressed by

$$
y= \pm \sqrt{\frac{c}{a\left(c^{2}-1\right)}} \sqrt{(u-m)^{2}(n-u)^{2}}
$$

where $-\sqrt{-e / 2 c}=m<u<n=\sqrt{-e / 2 c}$. Letting $u(0)=$ $(m+n) / 2=0$, we have

$$
\begin{gathered}
\int_{0}^{u} \sqrt{\frac{a\left(c^{2}-1\right)}{c}} \frac{d u}{(u-m)(n-u)}=\int_{0}^{\xi} d \xi, \quad-\infty<\xi<+\infty, \\
-\int_{0}^{u} \sqrt{\frac{a\left(c^{2}-1\right)}{c}} \frac{d u}{(u-m)(n-u)}=\int_{0}^{\xi} d \xi, \\
-\infty<\xi<+\infty .
\end{gathered}
$$

Noting that

$$
\int_{0}^{u} \frac{d u}{(u-m)(n-u)}=\frac{2}{n-m} \tanh ^{-1} \frac{2 u-(n+m)}{n-m},
$$

we obtain the corresponding shock wave solutions

$$
\begin{aligned}
& u_{1}(\xi)=\frac{n-m}{2} \tanh \left(\frac{n-m}{2} \sqrt{\frac{c}{a\left(c^{2}-1\right)}} \xi\right), \\
& u_{2}(\xi)=\frac{n-m}{2} \tanh \left(\frac{m-n}{2} \sqrt{\frac{c}{a\left(c^{2}-1\right)}} \xi\right),
\end{aligned}
$$

shown in Figure 8.

3.3. The Solitary Wave Solutions. In case $e / c<0$ and $e / a\left(c^{2}-\right.$ 1) $>0$, the homoclinic orbits $\gamma^{B}$ and $\gamma^{C}$ can be expressed by

$$
y= \pm \sqrt{-\frac{c}{a\left(c^{2}-1\right)}} \sqrt{(u+m) u^{2}(m-u)}
$$






(a)

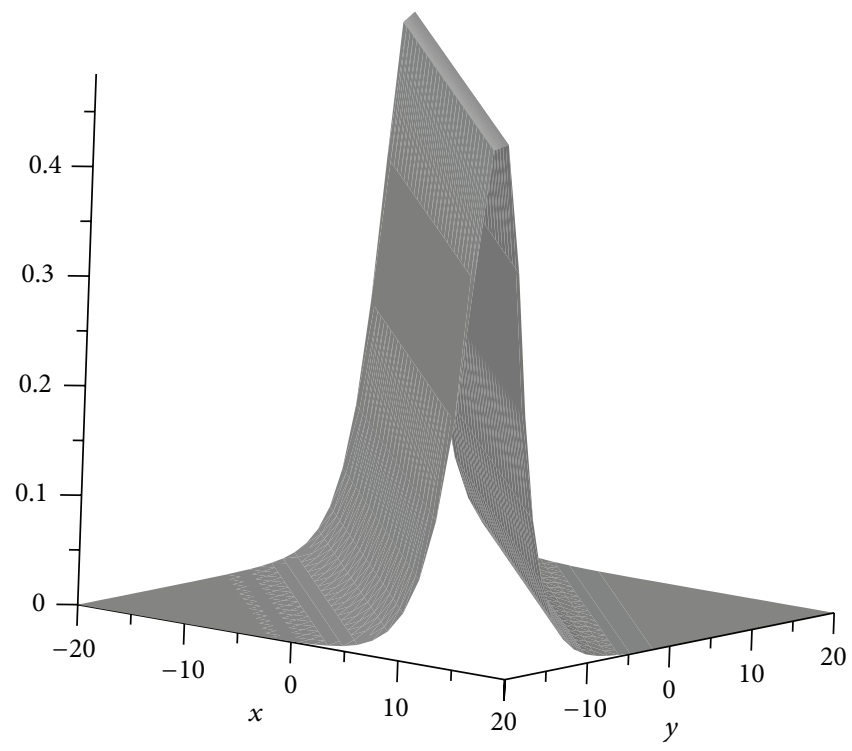

(b)

FIGURE 9: The solitary wave solutions.

where $-m<u<m$ and $m>0$. For homoclinic orbit $\gamma^{C}$, choosing $u(0)=-m$, we have

$$
\begin{array}{r}
\int_{-m}^{u} \sqrt{-\frac{a\left(c^{2}-1\right)}{c}} \frac{d u}{\sqrt{(u+m) u^{2}(m-u)}}=\int_{0}^{\xi} d \xi, \quad \xi>0, \\
-\int_{u}^{-m} \sqrt{-\frac{a\left(c^{2}-1\right)}{c}} \frac{d u}{\sqrt{(u+m) u^{2}(m-u)}}=\int_{\xi}^{0} d \xi, \\
\xi<0,
\end{array}
$$

which can be rewritten as

$$
\int_{-m}^{u} \sqrt{-\frac{a\left(c^{2}-1\right)}{c}} \frac{d u}{u \sqrt{(u+m)(m-u)}}=|\xi| .
$$

Noting that

$$
\int_{-m}^{u} \frac{d u}{u \sqrt{(u+m)(m-u)}}=\frac{\ln \left(\left(-m-\sqrt{m^{2}-u^{2}}\right) / u\right)}{-m},
$$

we get the expression of the corresponding solitary wave solution

$$
u(\xi)=\frac{-2 m \exp \left(\sqrt{-c / a\left(c^{2}-1\right)} m|\xi|\right)}{\exp \left(2 \sqrt{-c / a\left(c^{2}-1\right)} m|\xi|\right)+1}
$$

shown in Figure 9(a).
Similarly, for homoclinic orbit $\gamma^{B}$, choosing $u(0)=m$, we have

$$
\begin{aligned}
& \int_{u}^{m} \sqrt{-\frac{a\left(c^{2}-1\right)}{c}} \frac{d u}{\sqrt{(u+m) u^{2}(m-u)}}=\int_{\xi}^{0} d \xi, \quad \xi<0, \\
& -\int_{m}^{u} \sqrt{-\frac{a\left(c^{2}-1\right)}{c}} \frac{d u}{\sqrt{(u+m) u^{2}(m-u)}}=\int_{0}^{\xi} d \xi, \quad \xi>0,
\end{aligned}
$$

which can be rewritten as

$$
\int_{m}^{u} \sqrt{-\frac{a\left(c^{2}-1\right)}{c}} \frac{d u}{u \sqrt{(u+m)(m-u)}}=-|\xi| .
$$

Noting that

$$
\int_{m}^{u} \frac{d u}{u \sqrt{(u+m)(m-u)}}=\frac{\ln \left(\left(m-\sqrt{m^{2}-u^{2}}\right) / u\right)}{m},
$$

we get the expression of the corresponding solitary wave solution

$$
u=\frac{2 m \exp \left(\sqrt{-c / a\left(c^{2}-1\right)} m|\xi|\right)}{\exp \left(2 \sqrt{-c / a\left(c^{2}-1\right)} m|\xi|\right)+1}
$$

as shown in Figure 9(b).

\section{Discussion}

In this paper, we apply the dynamical system methods to investigate bounded traveling waves of the $(2+1)$-dimensional 
Zoomeron equation. This method allows detailed analysis on phase space geometry of the traveling wave system of the $(2+1)$-dimensional Zoomeron equation so that all possible bounded traveling waves and corresponding existence conditions can be identified clearly. We find that there exist lots of periodic waves besides solitary waves and shock waves for the $(2+1)$-dimensional Zoomeron equation. What is more, exact expressions of all bounded traveling waves of the $(2+1)$ dimensional Zoomeron equation are obtained. Among them, the periodic wave solutions can be expressed by the elliptic function uniformly and are new solutions.

\section{Conflict of Interests}

The authors declare that there is no conflict of interests regarding the publication of this paper.

\section{Acknowledgments}

This work is supported by the National Natural Science Foundation of China (no. 11301043, no. 11171046, and no. 61273007), the Key Project of Educational Commission of Sichuan Province (no. 12ZA224), and the Scientific Research Foundation of CUIT (no. J201219).

\section{References}

[1] F. Calogero and A. Degasperis, Spectral Transform and Solitons I, North-Holland, New York, NY, USA, 1982.

[2] R. Abazari, "The solitary wave solutions of Zoomeron equation," Applied Mathematical Sciences, vol. 5, no. 57-60, pp. 2943-2949, 2011.

[3] M. Alquran and K. Al-Khaled, "Mathematical methods for a reliable treatment of the $(2+1)$-dimensional Zoomeron equation," Mathematical Sciences, vol. 6, no. 1, article 11, 5 pages, 2012.

[4] A. Irshad and S. T. Mohyud-Din, "Solitary wave solutions for Zoomeron equation," Walailak Journal of Science and Technology, vol. 10, no. 2, pp. 201-208, 2013.

[5] A. Qawasmeh, "Soliton solutions of (2+1)-Zoomeron equation and Duffing equation and SRLW equation," Journal of Mathematical and Computational Science, vol. 3, no. 6, pp. 1475-1480, 2013.

[6] K. Khan and M. Ali Akbar, "Traveling wave solutions of the $(2+$ 1)-dimensional Zoomeron equation and the Burgers equations via the MSE method and the Exp-function method," Ain Shams Engineering Journal, vol. 5, no. 1, pp. 247-256, 2014.

[7] B. Katzengruber, M. Krupa, and P. Szmolyan, "Bifurcation of traveling waves in extrinsic semiconductors," Physica D: Nonlinear Phenomena, vol. 144, no. 1-2, pp. 1-19, 2000.

[8] J. B. Li and H. H. Dai, On the Study of Singular Nonlinear Traveling Wave Equation:Dynamical System Approach, Science Press, Beijing, China, 2007.

[9] D. Peterhof, B. Sandstede, and A. Scheel, "Exponential dichotomies for solitary-wave solutions of semilinear elliptic equations on infinite cylinders," Journal of Differential Equations, vol. 140, no. 2, pp. 266-308, 1997.

[10] F. Sánchez-Garduño and P. K. Maini, "Travelling wave phenomena in non-linear diffusion degenerate Nagumo equations," Journal of Mathematical Biology, vol. 35, no. 6, pp. 713-728, 1997.
[11] A. Constantin and W. Strauss, "Exact periodic traveling water waves with vorticity," Comptes Rendus Mathematique, vol. 335, no. 10, pp. 797-800, 2002.

[12] J. Huang, G. Lu, and S. Ruan, "Existence of traveling wave solutions in a diffusive predator-prey model," Journal of Mathematical Biology, vol. 46, no. 2, pp. 132-152, 2003.

[13] Y. Q. Zhou, Q. Liu, and W. N. Zhang, "Bounded traveling waves of the Burgers-Huxley equation," Nonlinear Analysis: Theory, Methods \& Applications, vol. 74, no. 4, pp. 1047-1060, 2011.

[14] Y. Zhou and Q. Liu, "Kink waves and their evolution of the RLW-burgers equation," Abstract and Applied Analysis, vol. 2012, Article ID 109235, 14 pages, 2012.

[15] J. Shi, S. Li, and J. Li, "Analytical travelling wave solutions and parameter analysis for the $(2+1)$-Dimensional daveyStewartson-Type equations," Pramana, vol. 81, no. 5, pp. 747762, 2013.

[16] J. B. Li and G. R. Chen, "Bifurcations of traveling wave solutions in a microstructured solid model," International Journal of Bifurcation and Chaos in Applied Sciences and Engineering, vol. 23, no. 1, Article ID 1350009, pp. 1-18, 2013.

[17] S. N. Chow and J. K. Hale, Method of Bifurcation Theory, Springer, New York, NY, USA, 1982.

[18] J. Guckenheimer and P. Holmes, Nonlinear Oscillations, Dynamical Systems and Bifurcation of Vector Fields, vol. 42 of Applied Mathematical Sciences, Springer, New York, NY, USA, 1983.

[19] J. K. Hale, Ordinary Differential Equations, John Wiley \& Sons, New York, NY, USA, 1997.

[20] Z. F. Zhang, T. R. Ding, W. Z. Huang, and Z. X. Dong, Qualitative Theory of Differential Equations, American Mathematical Society, Providence, RI, USA, 1992. 


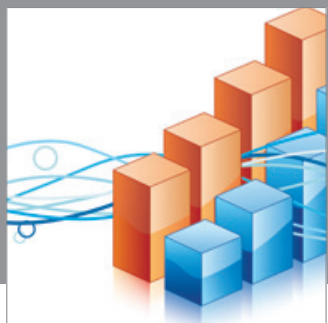

Advances in

Operations Research

mansans

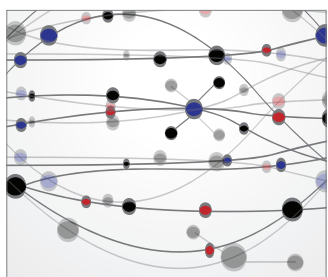

The Scientific World Journal
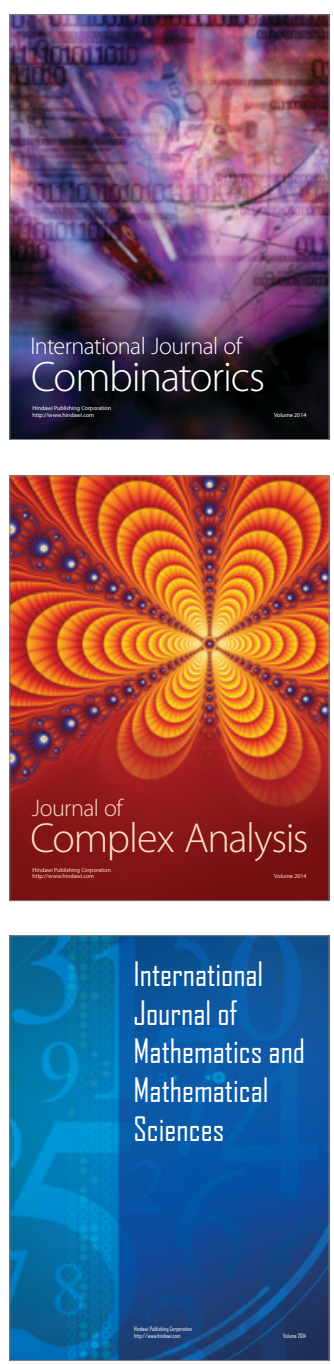
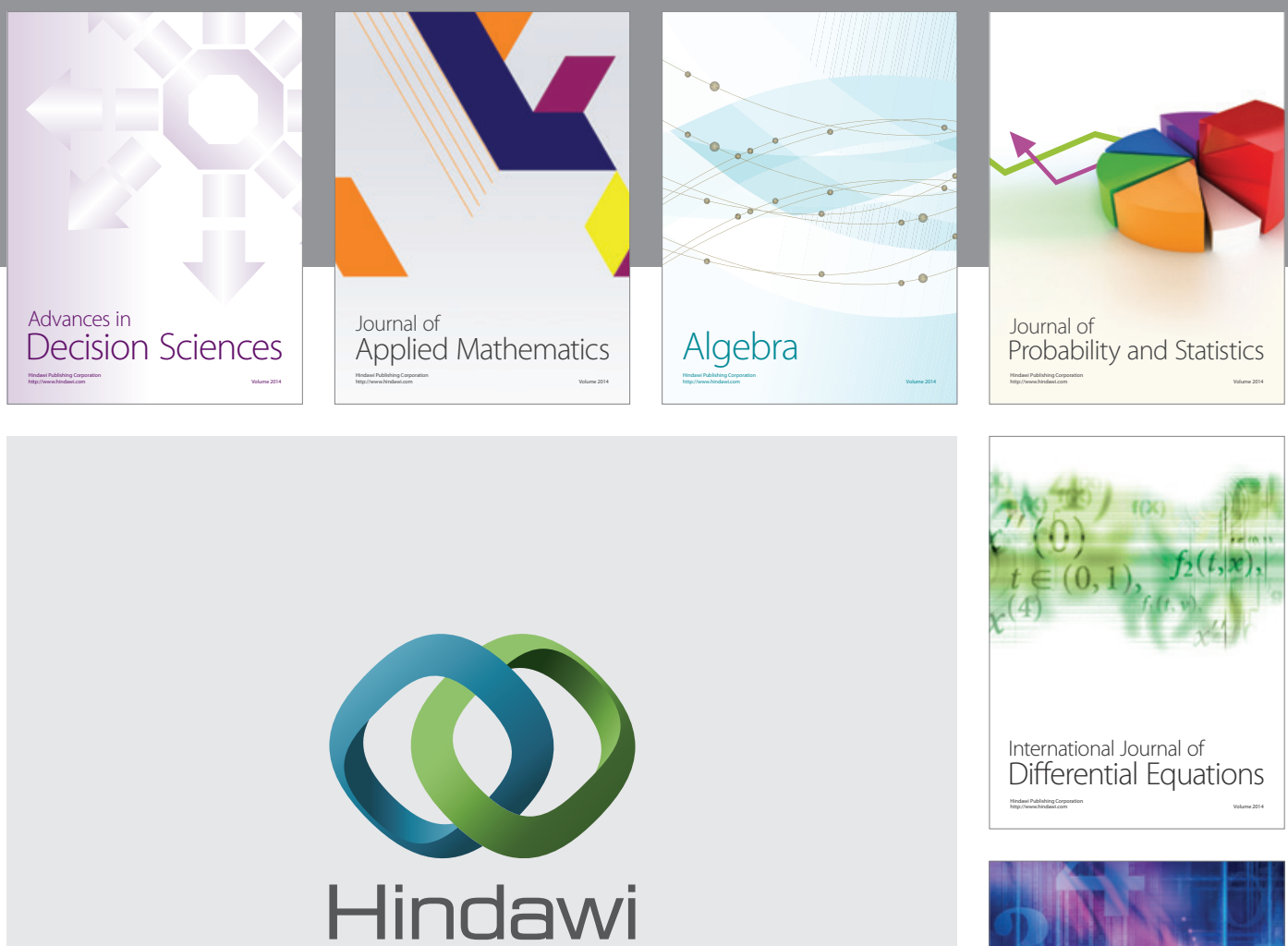

Submit your manuscripts at http://www.hindawi.com
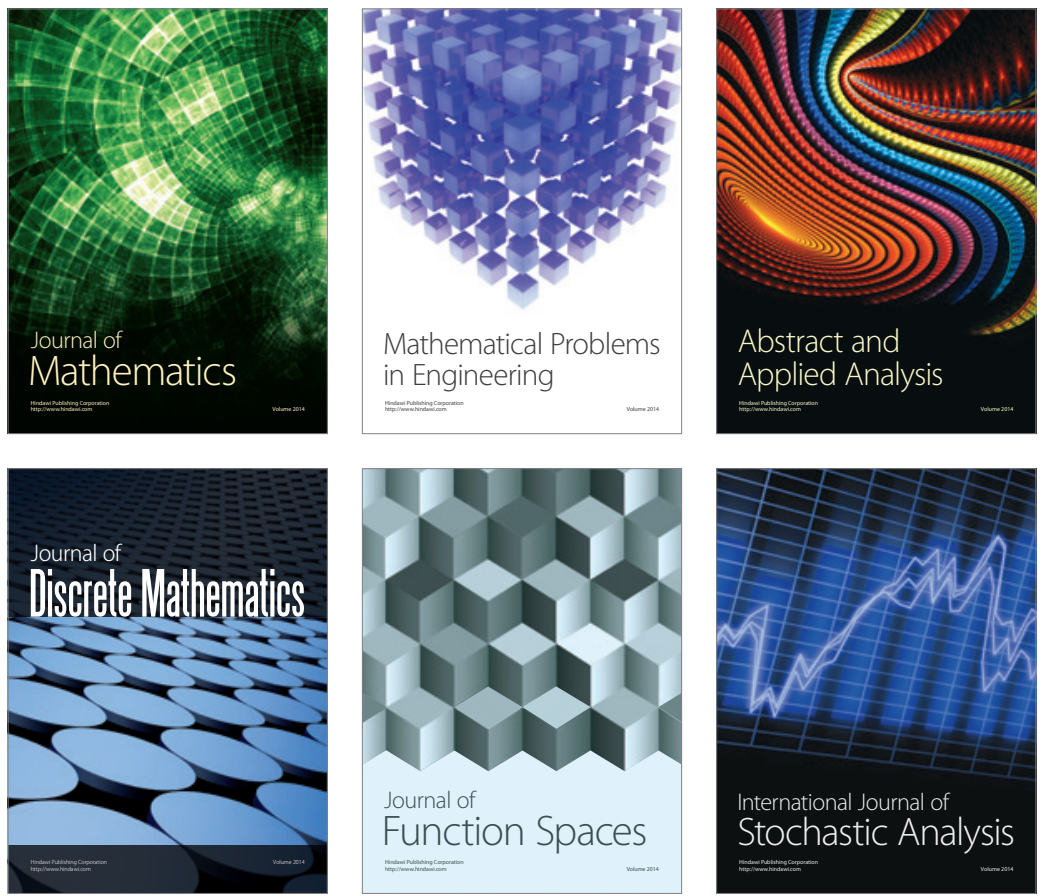

Journal of

Function Spaces

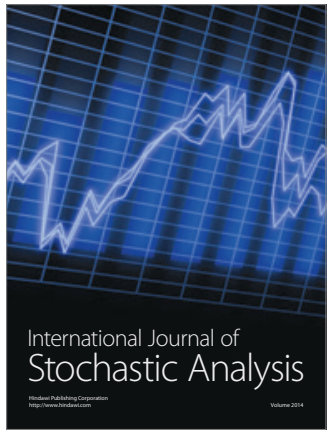

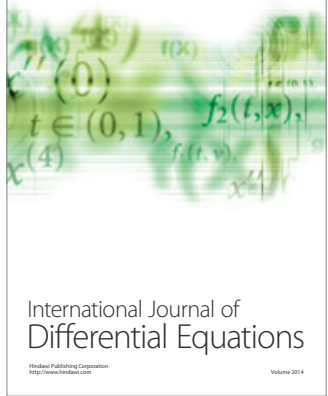
